\title{
Kinetics of Epidermal Growth Factor/Receptor Binding on Cells Measured by Total Internal Reflection/Fluorescence Recovery After Photobleaching
}

\author{
Edward H. Hellen ${ }^{1,2}$ and Daniel Axelrod ${ }^{1}$
}

Received June 13, 1991; accepted June 21, 1991

\begin{abstract}
Total internal reflection fluorescence (TIRF) microscopy is used to measure the dissociation kinetic rate of fluorescein-labeled epidermal growth factor from its specific receptors on the surface of intact but mildly fixed A431 human epidermoid cells in culture. Prior applications of TIRF microscopy have been limited to nonreceptor binding or to model membrane systems. The evanescent field excites fluorescence selectively at the surface of the cell proximal to the coverslip. "Prismless" epiillumination TIR is employed to avoid space limitations and is achieved by passing the excitation laser beam through a high (1.4)-aperture objective so that the light is incident at the glass/water interface beyond the critical angle. Long-term focus is maintained by a special feedback system. Of the possible effects that can influence the time course of the postbleach fluorescence recoveries-the EGF/receptor dissociation rate $k_{2}$, the bulk solution diffusion rate of EGF, and the cell surface motion of the receptors-we infer that the dissociation rate $k_{2}$ dominates. Several fitting schemes are compared and indicate the presence of a multiplicity of values for $k_{2}$, ranging from about 0.05 to $0.004 \mathrm{~s}^{-1}$, with an average value of about $0.012 \mathrm{~s}^{-1}$. These results compare well with values previously obtained by radiolabel/washing techniques. The significance of the results in terms of kinetic models and the advantages of the TIRF technique for these sorts of measurements are discussed.
\end{abstract}

KEY WORDS: Total internal reflection; fluorescence; membranes; hormones; receptors.

\section{INTRODUCTION}

Epidermal growth factor (EGF) is an extensively studied hormone which is mitogenic for a variety of cell types [1-3]. However, the mechanism by which EGF activates cellular responses is not known. Elucidation of the mechanism may be aided by accurate knowledge of the dissociation rate constant characterizing the interaction of EGF with its receptor molecule on cell surfaces. The reciprocal of the dissociation rate constant is the characteristic time that the hormone stays bound to the

${ }^{1}$ Department of Physics and Biophysics Research Division, University of Michigan, Ann Arbor, Michigan 48109.

${ }^{2}$ To whom correspondence should be addressed at Department of Biochemistry and Molecular Biology, Mayo Foundation, Rochester, Minnesota 55905. receptor at the cell surface before unbinding. Comparison of this binding time to the delay between hormone exposure and various cellular responses can, in principle, help distinguish between different proposed mechanisms of activation. This paper demonstrates the use of total internal reflection/fluorescence recovery after photobleaching (TIR/FRAP) [4] to measure the dissociation rate constant of EGF interacting with its receptor on A431 cells in culture.

\section{TIR/FRAP and Binding Kinetics at Surfaces}

In TIR/FRAP, fluorescent molecules in a liquid within about $100 \mathrm{~nm}$ of a solid surface are selectively excited by the exponentially decaying evanescent field of a laser beam that totally internally reflects at the solid/ 
liquid interface [5]. A bright brief flash of evanescent light bleaches some of the surface-proximal fluorophores; subsequent recovery of fluorescence excited by a much attenuated evanescent field arises from kinetic exchange with unbleached molecules in the bulk liquid.

The main purposes of using TIR for illumination of the A431 cells are (1) to selectively excite (and photobleach) fluorescent-labeled EGF bound to the cell surface near cell/substrate contacts, rather than free EGF dissolved in the bulk, and (2) to reduce the contribution of cell autofluorescence, most of which would otherwise arise from regions deeper within the cell and out of range of the evanescent TIR field.

Previous applications of TIR/FRAP have measured equilibrium and kinetic constants for either specific binding only on model systems or nonspecific binding. Burghardt and Axelrod [6] demonstrated that TIR/FRAP can be used to measure the dissociation rate constant(s) and surface diffusion of bovine serum albumin (BSA) nonspecifically and reversibly adsorbed to a glass surface. Tilton et al. [7] used TIR/FRAP to measure surface diffusion of BSA irreversibly bound to polymer coated glass. TIR fluorescence microscopy has been used to measure equilibrium constants of antibodies binding to Fc receptors [8] and to lipid haptens [9] in substrate-supported planar membranes. Zimmermann et al. [10] used TIR/ FRAP to quantitate surface concentration and measure dissociation kinetics of BSA interacting with plain quartz and alkylated quartz. Fulbright [11] has measured the dissociation rate of f-insulin interacting nonspecifically with red blood-cell ghosts. In contrast to these previous investigations, this paper describes an application of TIR/ FRAP for measuring the dissociation rate constant of a specific interaction between ligand (EGF) and its cell surface receptor, on actual cells (A431) in culture.

\section{EGF/Receptor Binding Kinetics}

EGF is a polypeptide hormone of molecular weight 6045. Receptors specific for EGF have been demonstrated on a variety of cells including fibroblasts and epithelial cells [1]. EGF induces numerous cellular responses. Initially these responses include increases in $\mathrm{Na}^{+} / \mathrm{H}^{+}$exchange, calcium transport, and phosphorylation of a variety of substrates. Long-term (10- to 12 hr) exposure to EGF leads to DNA replication and cell proliferation [1-3].

The EGF receptor is a 170 -kilodalton $(\mathrm{kD})$ integral membrane glycoprotein with a single transmembrane portion of 23 amino acids which connects the extracellular EGF binding domain (621 amino acids) to the biochemically active cytoplasmic domain (542 amino acids)
[12]. The cytoplasmic portion contains a tyrosine specific protein kinase and several autophosphorylation sites. Activation of the kinase appears to be necessary for signal transduction leading to mitogenesis [2]. Yet the mechanism by which EGF binding activates the receptor's kinase activity is not known. The TIR/FRAP measurement of the dissociation rate constant presented here may be useful to the understanding of this mechanism.

There are various kinetic models for the interaction of EGF with its receptor. One model claims that the receptor can exist in two affinity states, with 5 to $10 \%$ of the receptors in the higher-affinity state $[2,13-15]$. Other models claim that there is only one affinity state of the receptor but that other processes must be included, such as internalization of hormone-receptor complexes, down regulation of receptor, or formation of a ternary complex out of the hormone-receptor complex and an unidentified interaction protein [16-19].

For the purposes of fitting data we use two models: a single-affinity state model and a two-affinity state model. In the first model we assume that internalization of receptors is inhibited and that there is a single affinity state of the receptor. Therefore, this model for the interaction of EGF with its receptor is

$$
E G F+R \underset{k_{2}}{\stackrel{k_{1}}{\rightleftharpoons}} E G F-R
$$

or, in general,

$$
A+B \underset{k_{2}}{\stackrel{k_{1}}{\rightleftharpoons}} C
$$

where $k_{1}$ is the association rate constant with units of $M^{-1} \mathrm{~s}^{-1}$ and $k_{2}$ is the dissociation rate constant with units of $\mathrm{s}^{-1}$. EGF $(A)$ is the concentration of EGF in solution, $R(B)$ is the surface concentration of unoccupied receptors, and $E G F-R(C)$ is the surface concentration of occupied receptors. The equilibrium dissociation constant, $K_{\mathrm{D}}=k_{2} / k_{1}$, gives the ligand concentration at which half of the receptors are occupied.

In the two-affinity state model we assume that there are two noninteracting populations of binding sites, each with its own set of $k_{1}, k_{2}$, and $K_{\mathrm{D}}$.

EGF binds with a high affinity to receptors at the cell surface. Reported values for $K_{\mathrm{D}}$ are in the nanomolar range $[1,12,16,18,20]$. Within a few minutes it is internalized via receptor-mediated endocytosis and subsequently degraded through lysosomal action [21].

${ }^{125}$ I-Labeled binding assays have been used to measure EGF's dissociation rate constant (see Table I). In general, $k_{2}$ is in the range of $10^{-2}$ to $10^{-4} \mathrm{~s}^{-1}$, and the 
Table I. Reported Dissociation Rate Constants from ${ }^{125}$ I-EGF Binding Assays

\begin{tabular}{lllr}
\hline Reference & \multicolumn{1}{c}{ Binding substrate } & $k_{2}\left(\mathrm{~s}^{-1}\right)$ & $1 / k_{2}(\mathrm{~s})$ \\
\hline Knauer et al. [16] & Human fibroblast & 0.012 & 83 \\
Wiley [17] & A431 membrane prep. & 0.0018 & 560 \\
& Human fibroblast & 0.0036 & 280 \\
Mayo et al. [18] & Human fibroblast & $0.0049^{a}$ & 200 \\
& & $0.015^{b}$ & 67 \\
Waters et al. [19] & Fetal rat lung & 0.002 & 500 \\
Bellot et al. [2] & Transfected NIH 3T3 & 0.0003 & 3000 \\
\hline
\end{tabular}

${ }^{a}$ From single state model.

${ }^{b}$ From tertiary complex model.

rate of internalization $k_{\mathrm{e}}$ for EGF on human fibroblast cells is about $0.002 \mathrm{~s}^{-1}[16,19]$. The wide variations among the published results with ${ }^{125} \mathrm{I}$ and the significant differences between the ${ }^{125} \mathrm{I}$ binding assay technique and TIR/FRAP make the two techniques complementary. Distinguishing features of TIR/FRAP are that there is no washing of cells, chemical equilibrium is maintained, smaller time resolution is possible, multiple measurements can be made on the same sample, and measurements can be made from a portion of a single cell.

Comparison of $k_{2}$ and $k_{\mathrm{e}}$ determines whether a particular EGF is likely to be internalized during its first successful encounter with a receptor. Comparison of $k_{2}$ with the characteristic rate $k_{\mathrm{bd}}$ at which bulk diffusion can supply EGF to the receptors (to be defined precisely later) determines the average number of times a particular EGF binds and unbinds to receptors while in the vicinity of the surface. A goal of this work is to use the novel technique of TIR/FRAP to provide independent measurements of $k_{2}$ and $k_{\mathrm{bd}}$.

\section{Experimental Considerations}

It is desirable to maximize the signal due to specific binding by choosing a cell which has a large number of receptor molecules at its surface. Thus we chose the A431 cell (a human epidermoid cell line) since these cells are known to have an unusually high number of EGF receptors at their surface, about 2 million per cell. In contrast, most cells which respond to EGF contain 40,000 to $100,000 \mathrm{EFG}$ receptors per cell [22].

We use a new method of achieving TIR in order to avoid certain space limitations of the standard method. In standard TIR fluorescence microscopy, the incident laser beam first traverses through a prism in optical contact with the glass slide or coverslip that forms the TIR surface [5]. The fluorescence then travels through the liquid toward the objective, usually located behind a second glass coverslip. The thickness of the liquid layer between the coverslips, determined by a spacer, thereby limits the minimum working distance allowed for the objective. To avoid this restriction we used a prismless TIR technique (described in Appendix 1) similar in principle to that described by Stout and Axelrod [23] but simpler in practice (see Appendix 1). In this technique, the high-aperture (1.4) objective serves simultaneously to cast the incident beam upon the surface at greater than the critical angle and to gather the fluorescence; this is essentially "epiillumination" with TIR. Unlike standard TIR, this system is compatible with any depth of liquid, making it more convenient for cell culture work. The prismless TIR study described here is the first application of this technique beyond the demonstrations of Stout and Axelrod [23].

A recently introduced automated system for keeping samples in focus on a microscope was used for the TIR/FRAP experiments. This automatic focus/hold system is described by Hellen and Axelrod [24].

\section{MATERIALS AND METHODS}

\section{Cell Culture}

A431 cells purchased from American Type Culture Collection (Rockville, MD) were grown in Corning 25$\mathrm{cm}^{2}$ tissue culture flasks at $37^{\circ} \mathrm{C}$ under $7 \% \mathrm{CO}_{2}$. The medium was $90 \% 4.5 \mathrm{~g} /$ liter glucose DMEM, $10 \% \mathrm{FCS}$, and $0.5 \%$ penicillin-streptomyosin. Cells were replated about every 7 days using $0.25 \%$ trypsin with $0.02 \%$ versene. All cell culture materials were purchased from GIBCO. For experiments, cells were replated into 35$\mathrm{mm}$ glass bottom petri dishes (GBPD). These were constructed by using a circular saw to cut holes in the bottom of standard plastic petri dishes and then gluing in glass coverslips with RTV silicone sealant.

\section{Fluorescein-Labeled EGF}

Murine EGF was purchased from Biomedical Technologies, Inc. (Stoughton, MA). It was labeled using fluorescein isothiocyanate (FITC) purchased from Research Organics (Cleveland, $\mathrm{OH}$ ) using the following protocol, a slight variation of one suggested by Dr. David Johnson (UC-Riverside).

One hundred micrograms of lyophilized EGF was dissolved in $200 \mu$ l dimethylformamide (DMF). About $1 \mathrm{mg}$ of FITC dissolved in $50 \mu \mathrm{l} \mathrm{DMF}$ was added, followed by $50 \mu \mathrm{l}$ of pyridine. The solution was stirred in 
the dark for $4 \mathrm{~h}$. Fluorescein-labeled EGF (f-EGF) was separated from free dye by running the solution down a $20 \times 1-\mathrm{cm}$ diameter Sephadex G-15 column equilibrated with $10 \mathrm{mM}$ acetate, $\mathrm{pH}$ 5.6. A peristaltic pump ran the column at about $1 \mathrm{ml} / \mathrm{min}$. Fractions were collected and analyzed for EGF and fluorescein by fluorescence emission spectroscopy. Control experiments showed that excitation at $280 \mathrm{~nm}$ with emission at $350 \mathrm{~nm}$ could be used to detect protein and excitation at $490 \mathrm{~nm}$ with emission at $515 \mathrm{~nm}$ could be used to detect fluorescein. The concentration of the f-EGF fraction was $7 \mu M$ determined by the Pierce (Rockford, IL) bicinchoninic acid protein assay, enhanced protocol.

Trial G-15 columns were run under the same conditions as the labeling protocol in order to determine how much free dye might elute with the EGF. Unlabeled EGF and fluorescein were separately run down the column. Fluorescence emission spectroscopy showed clear separation between the (early) EGF peak and the (later) broad fluorescein band. When the actual labeling solution was run, the fluorescein label showed two peaks, one coinciding with the EGF at the expected position and a much larger free dye peak appearing later.

Butanol extractions performed on $\mathrm{f}$-EGF fractions from the G-15 column showed that only a small fraction of the fluorescein went into the butanol. Control butanol extractions showed that nearly all free fluorescein goes into the butanol and that hormone stays in the aqueous phase.

\section{Sample Preparation}

For TIR/FRAP experiments, a GBPD with healthylooking cells was used 4 to 7 days after replating. The GBPD was placed in a custom-built chamber described in the next section. In all cases, cells were first washed in Hank's balanced salt solution (HBSS) or Dulbecco's phosphate-buffered saline (DPBS). The buffer for the experiment was $3 \mathrm{ml}$ of DPBS or HBSS and was partially deoxygenated by bubbling nitrogen or argon through it before addition to the cells. In approximately half the experiments the buffer contained bovine serum albumin at concentrations of $1-5 \mathrm{mg} / \mathrm{ml}$. F-EGF concentrations were in the 1-100 $\mathrm{n} M$ range, with most about $5 \mathrm{n} M$.

Three methods were used to inhibit ligand-induced receptor internalization: cooling [22], fixing [14,21], and addition of metabolic poisons [25]. For experiments on cooled cells, f-EGF was not added to the cells until after they had been cooled by a thermoelectric Peltier junction attached to the sample chamber (described in the section below). For experiments on fixed cells, the cells were exposed to 0.2 or $1 \%$ formaldehyde in DPBS for $5-10$ min. The cells were then washed again before adding the experimental buffer and f-EGF. For experiments using metabolic poisons, the DPBS buffer was augmented with $20 \mathrm{~m} M$ 2-deoxyglucose and $3 \mathrm{~m} M$ sodium azide; poisoned cells were also cooled in some experiments. F-EGF was added after exposure to the poisons and the cooling.

In control experiments used to investigate accessibility of cell surface to hormone, fluorescently labeled insulin (Sigma) was used at concentrations of 40 and $200 \mu M$ instead of f-EGF. In control experiments used to investigate the effect of lateral diffusion of receptors, cells were fixed for $30 \mathrm{~min}$ in $4 \%$ formaldehyde, followed by washing, then $20 \mathrm{~min}$ in $2 \mathrm{mg} / \mathrm{ml} \mathrm{BSA}$ in DPBS prior to the addition of $\mathrm{f}-\mathrm{EGF}$.

\section{Sample Chamber}

A custom-built sample chamber was used to hold the GBPD on the microscope stage. This chamber provides an airtight seal which is necessary to keep the sample partially deoxygenated. Ports with valves allow for the addition of $\mathrm{f}$-EGF to the sample and for further bubbling of nitrogen or argon to remove oxygen. The top of the chamber is aluminum with a peltier junction attached. (Peltier junction and power supply from Bailey Instruments, Saddle Brook, NJ.) Two stainless-steel nuts extend into the buffer providing cooling. A window in the top allows for transmitted light illumination.

\section{Optics}

The laser beam for fluorescence illumination ("probe") and photobleaching was provided by the 488$\mathrm{nm}$ line of a 3-W Lexel argon laser. An acoustooptic modulator (NEC Corporation) controlled the laser intensity for illumination and photobleaching. The bleach-toillumination ratio was about 4000:1, and the probe illumination level at the sample was about 10 microW. The size of the illuminated region was approximately $250 \mu \mathrm{m}^{2}$.

Figure 1 is a diagram of the apparatus. The inverted epifluorescence microscope (Leitz Diavert), outfitted with a dichroic mirror and barrier filter appropriate for fluorescein, employed an oil immersion $60 \times, 1.4$ numerical aperture Nikon objective.

Mirrors direct the beam to be at a slight angle with respect to the optical axis so that it will propagate just inside the periphery of the 1.4 aperture objective and emerge from the objective to impinge on the sample plane at greater than the critical angle for total internal reflection. To ensure that the beam is reasonably colli- 


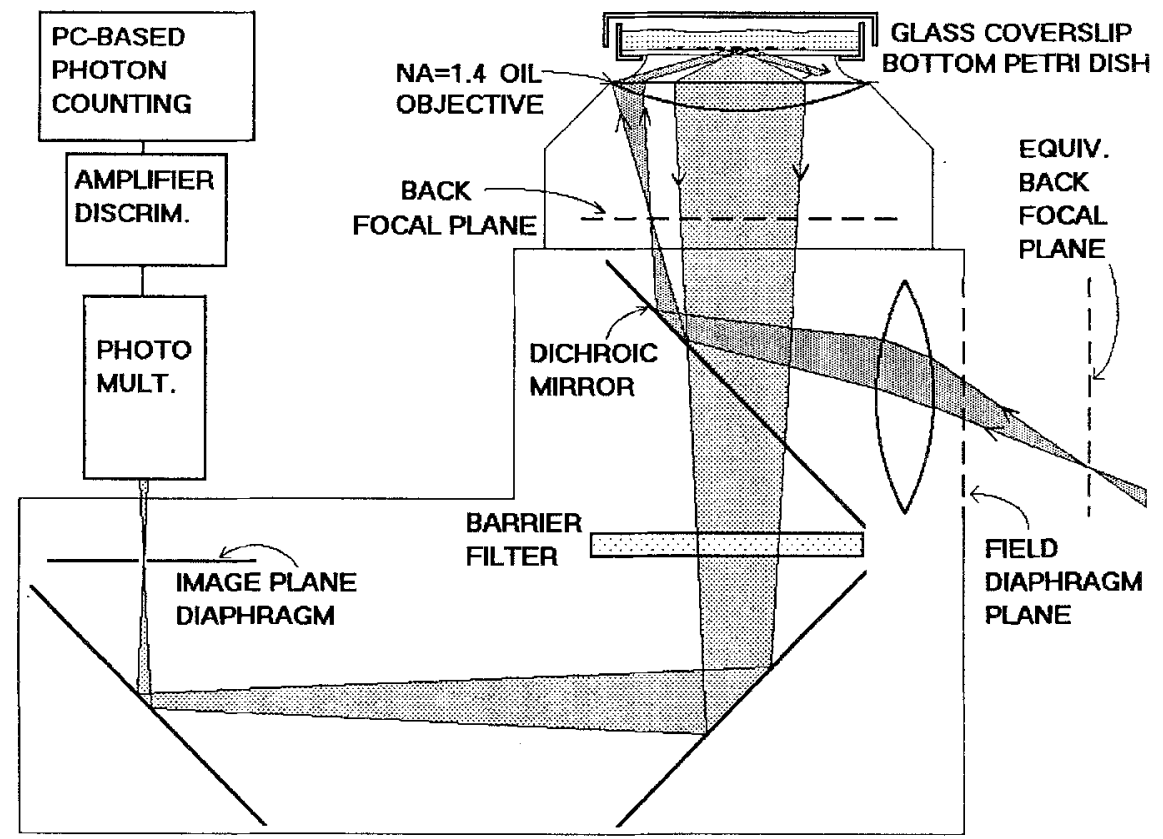

Fig. 1. Diagram of the apparatus used for exciting and collecting fluorescence in the TIR/FRAP experiments. TIR excitation is accomplished by passing the laser beam through the periphery of a 1.4 numerical aperture objective as explained in Appendix 1. Fluorescence (light stipple) is gathered by the same objective and passed through the microscope to a photomultiplier tube interfaced to a computer. The incident laser beam (dark stipple) propagates in planes perpendicular to the plane of the diagram, but for clarity, it is shown in the plane of the diagram. The size scales and angles, particularly in the greatly enlarged objective region, are distorted for pictorial clarity.

mated as it approached the sample plane, it must be focused at the objective's back focal plane. This can be accomplished simply by ensuring that the beam is focused at the "equivalent" back focal plane located (for our microscope) at about $10 \mathrm{~cm}$ upbeam from the microscope's field diaphragm.

Fluorescence intensity was measured with a thermoelectrically cooled photomultiplier tube (PMT, Hamamatsu R943-02) connected to a commercial amplifier/ discriminator (Model 3470/AD6, Pacific Instruments Inc) operating in photon counting mode. The PMT was protected from excessive count rate during the photobleaching pulse by electronically shorting the first dynode of the photocathode during that pulse only.

\section{Electronics}

An AT-compatible computer (Zenith-248) was used for data collection and control of the acoustooptic modulator in the FRAP experiments. The photon pulses from the PMT went to an amplifier/discriminator operating in photon counting mode which was interfaced to a rate meter, a strip-chart recorder, and a PC-compatible counter/ timer (Metrabyte CTM-05) installed in the computer.
Experimental parameters were input to a custom-made Fortran program which controlled sample time, number of time intervals, bleaching pulse, PMT protection, and storage of counts per sample time in memory.

\section{TIR/FRAP Protocol for f-EGF on Cells}

The evanescent wave extends only far enough into the water to excite fluorescence at the portion of the cell membrane adjacent to the coverslip. Figure 2 schematically shows a region from which fluorescence was typically collected. An image plane diaphragm was set to collect fluorescence from about a quarter of the portion of a single cell's membrane adjacent to the coverslip. Only cells at the periphery of patches of cells (i.e., adjacent to bare glass) were selected, and the selected region of membrane was next to the bare glass. To subtract any luminescence contribution from the bulk solution or glass, control experiments were done with identical optics on adjacent bare glass regions. In general, this background contribution was not significant.

Before starting a TIR/FRAP run at any location, fluorescence was monitored to determine that it was constant. Typically $2 \mathrm{~min}$ of prebleach fluorescence and 8 


\section{A431 Cells on Coverslip}

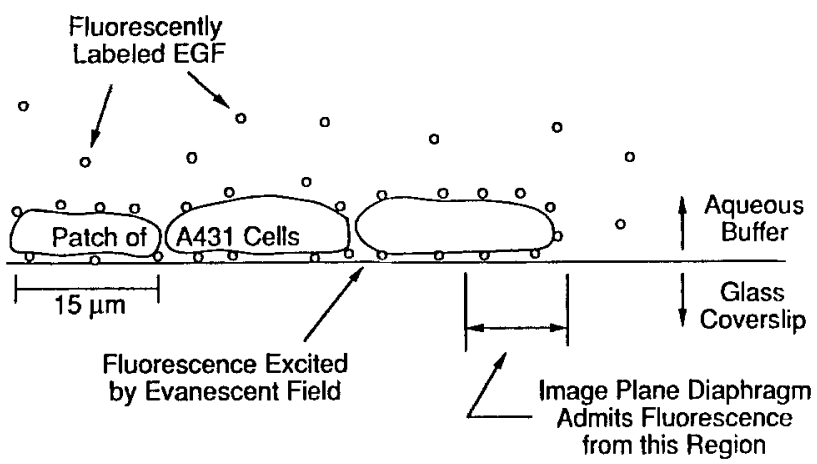

Fig. 2. Diagram showing the region from which fluorescence was collected. The sample was positioned so that the image plane diaphragm collected fluorescence from a portion of one cell next to bare glass. The cells are grown using standard cell culture procedures in glass coverslip-bottom petri dishes which, after replacing the growth medium with the appropriate buffer, are placed directly on the microscope stage for TIR/FRAP experiments.

to $12 \mathrm{~min}$ of postbleach fluorescence were recorded. This was accomplished using 1000 sample times, each of length 0.6 or $0.84 \mathrm{~s}$. Bleach pulse durations were 20,50 , or $100 \mathrm{~ms}$. Background fluorescence was estimated by monitoring the fluorescence signal from a cell sample with no f-EGF.

Control experiments using f-insulin (at 5 to $10 \mathrm{n} M$ ) or free FITC (at 10 to $50 \mathrm{nM}$ at $\mathrm{pH} \mathrm{7.4)} \mathrm{in} \mathrm{place} \mathrm{of}$ f-EGF were performed similarly to the f-EGF experiments described above.

\section{Chasing and Blocking Experiments}

In chasing experiments, an excess of unlabeled EGF was added to cells that had been previously prepared with $\mathrm{f}-\mathrm{EGF}$ using the conditions for TIR/FRAP experiments, while maintaining the original bulk concentration of $\mathrm{f}$-EGF. The concentration of unlabeled EGF $(\approx 500$ $\mathrm{n} M)$ was more than $50 \times$ the concentration of $\mathrm{f}-\mathrm{EGF}(1-$ $10 \mathrm{nM}$ ). Typically, the chase was done after some TIR/ FRAP runs had been done. Fluorescence intensity of cells and bare glass before and after the chase was compared. In addition, fluorescence recovery magnitudes from TIR/FRAP experiments performed before and after the chase were compared.

In blocking experiments, an excess of unlabeled EGF was added to the cells first. The cells then were observed on the microscope, and in some cases TIR/
FRAP runs were done. Subsequently, f-EGF was added at the concentrations used in unblocked TIR/FRAP runs.

Chasing and blocking control experiments were also performed using unlabeled insulin (Sigma) in place of unlabeled EGF.

\section{Curve Fitting}

A hybrid gradient search/analytical solution routine from Bevington [26] was used to curve fit the fluorescence recovery data. Three different fitting functions were used, to be discussed under Results. In theory, the time dependence of the fluorescence recovery curve is determined by the dissociation rate constant $k_{2}$ of the binding and by a characteristic bulk diffusion time (defined under Results) of ligand in solution. The different fitting functions arise from different assumptions about the relative size of these rates and the heterogeneity of the sample with regard to dissociation rates.

\section{RESULTS}

\section{f-EGF on A431 Cells}

Relative concentrations of bound $\mathrm{f}-\mathrm{EGF}$ as a function of bulk concentration of $\mathrm{f}$-EGF was estimated by comparing fluorescence intensities of cells. Cell-to-cell variations made only rough estimates possible. At 5-10 $\mathrm{n} M$, the concentration used in most of the TIR/FRAP runs, fluorescence intensity was at roughly half its maximal level. Around $20 \mathrm{n} M$, the fluorescence had reached its maximal level.

Strip-chart records of approximately 250 TIR/FRAP runs (collected from 24 days spread over 8 months) were examined. Runs in which prebleach fluorescence $F_{\text {pre }}$ or the $t \rightarrow \infty$ fluorescence $F(\infty)$ were clearly not constant were rejected. The remaining 172 runs include a variety of f-EGF concentrations and cell preparation protocols. These runs were curve fit using three different fitting functions.

Table II shows the results from the TIR/FRAP data which was curve fit as described below. The results from the various methods of inhibiting internalization showed no obvious differences and are grouped together in Table II. Standard deviations from the mean (rather than "standard errors" of the mean) are shown because the variabilities in the data may represent an actual range in biology rather than random uncertainties of statistics. 
Table II. Curve-Fitting Results for Kinetic Parameters ${ }^{a}$

\begin{tabular}{|c|c|c|c|c|}
\hline Single exponential & $\begin{array}{c}t_{\mathrm{off}}=1 / k_{2} \\
(\mathrm{~s})\end{array}$ & $\%$ recovery & & \\
\hline All runs $(172)$ & $135(55)$ & $73(15)$ & & \\
\hline Selected (134) (see text) & $145(53)$ & $73(15)$ & & \\
\hline Low conc. (17) & $233(65)$ & $80(12)$ & & \\
\hline Mid conc. (27) & $152(53)$ & $78(14)$ & & \\
\hline High conc. (31) & $125(47)$ & $71(14)$ & & \\
\hline Room temp. (19) & $156(44)$ & $80(14)$ & & \\
\hline Cooled (8) & $145(67)$ & $73(11)$ & & \\
\hline Double exponential & $\begin{array}{c}1 \mathrm{st} t_{\mathrm{off}}=1 / k_{2} \\
(\mathrm{~s})\end{array}$ & $\%$ recovery & 2 nd $t_{\text {off }}=1 / k_{2}$ & $\%$ recovery \\
\hline All runs (137) & $20(12)$ & $27(11)$ & $250(125)$ & $60(17)$ \\
\hline Exact & $\begin{array}{c}t_{\text {off }}=1 / k_{2} \\
(\mathrm{~s})\end{array}$ & $\%$ recovery & $\begin{array}{c}t_{\mathrm{bd}}=l^{2} / 4 D \\
(\mathrm{~s})\end{array}$ & \\
\hline All runs $(103)$ & $61(55)$ & $97(26)$ & $33(52)$ & \\
\hline Selected (75) (see text) & $87(39)$ & $90(22)$ & 19 (27) & \\
\hline Low conc. (5) & $149(42)$ & $102(44)$ & $61 \quad(59)$ & \\
\hline High conc. (21) & $82(38)$ & $81(17)$ & $15 \quad(26)$ & \\
\hline
\end{tabular}

aThe number of runs in each set is given in parentheses in the first column. Standard deviations are given in parentheses following each value.

\section{Single-Exponential, Reaction-Limit Fitting Function}

Each of the 172 fluorescence recovery curves was curve fit using the single-exponential fitting function:

$$
F(t)=A_{1}-A_{2} e^{-A_{3 t}}
$$

This function is appropriate for the case where all the receptors exhibit the same kinetic behavior, and the process is reaction rate limited (i.e., the reaction rates at the surface are slow enough so that bulk diffusion to the surface is not rate limiting; see Exact Fitting Function below.). Parameter $A_{1}$ represents the final fluorescence $F(\infty), A_{2}$ is the magnitude of the recovery, and $A_{3}$ is the dissociation rate constant $k_{2}$. All three $A_{i}$ 's are nonnegative free parameters. The percentage recovery of fluorescence is definded as $A_{2}$ / $\left(F_{\text {pre }}-A_{1}+A_{2}\right)$. Average values for $1 / k_{2}$ and percentage recovery are shown in Table II. Of the 172 fits, 44 had reduced chi-square values greater than 1.5 . Figure 3 shows the fluorescence data and a curve fit from the single-exponential fit for a typical TIR/FRAP run (along with an "exact fit" discussed under Exact Fitting Function below).

Two types of runs were removed from the set of 172 runs: "small amplitude" runs and "bleaching dur- ing illumination" runs. For these purposes the amplitude of a run is defined to be the ratio of the fluorescence recovery amplitude to the final fluorescence, $A_{2} / A_{1}$. The $k_{2}$ values for runs with low amplitudes are more susceptible to influence from drifting fluorescence levels. Therefore, those runs with $A_{2} / A_{1}<0.15$ were removed from the 172 runs. In addition, some runs had significant photobleaching during illumination ("significant" defined to be when the fluorescence after tens of minutes of constant probe illumination falls to less than $80 \%$ of the initial fluorescence). Appendix 2 shows that bleaching during illumination increases the fluorescence recovery rate. Therefore, these runs were also removed from the 172 runs, leaving 134 runs in the first "selected" set in Table II.

Dependence of recovery rate on f-EGF concentration was checked by separately collecting sets of runs at high concentrations ( $\geq 10 \mathrm{nM}$ ), at midconcentrations (5$10 \mathrm{n} M$ ), and at low concentrations ( $\leq 5 \mathrm{n} M$ ) among the group of 134 runs. The recovery rate at even higher concentrations than the above "high concentration" set was measured in runs in which $\mathrm{f}$-EGF was added to a sample between runs. The concentration was increased fivefold, starting at a concentration $(\approx 20 \mathrm{n} M)$ in the above "high-concentration" set. The recovery rate in 


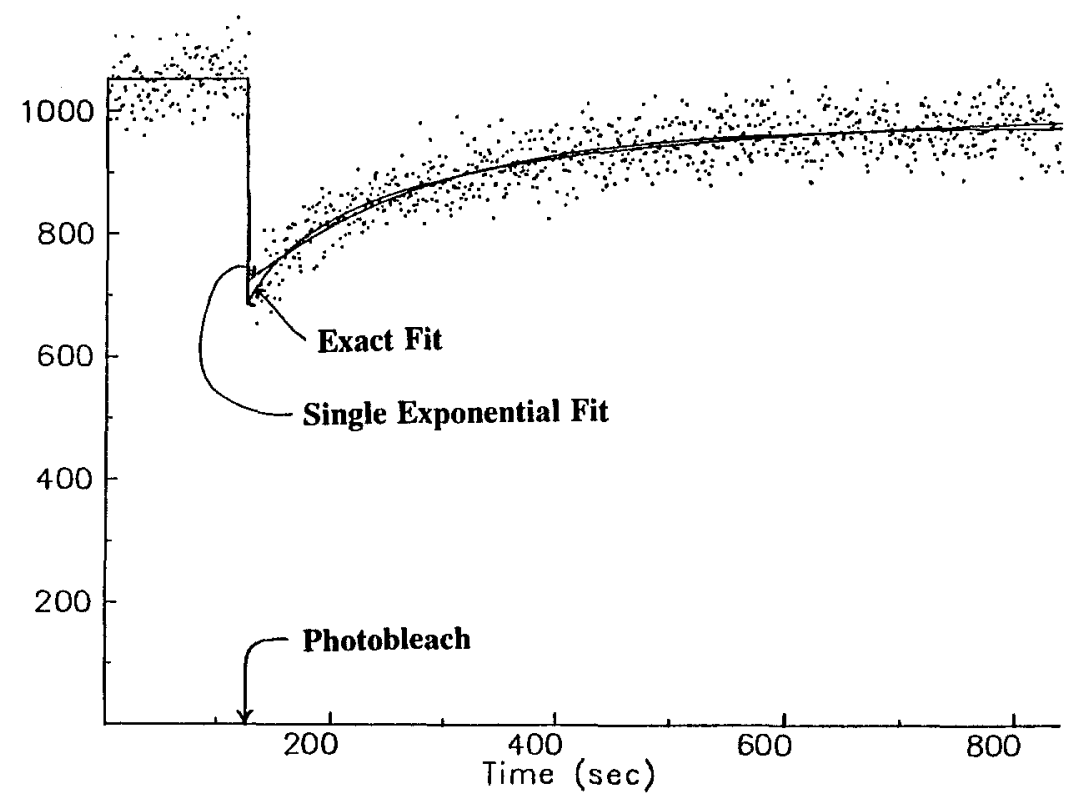

Fig. 3. Data from a single TIR/FRAP run (dots) and two curve fits, the single-exponential fit and the exact fit, of the data. The single-exponential curve fit is the higher of the two right after the photobleach and is the lower of the two at $t=840 \mathrm{~s}$. The exact fit follows the data more closely than the single-exponential fit. The photobleaching pulse was $100 \mathrm{~ms}$ long and occurred at $t=126 \mathrm{~s}$.

this set of seven runs showed no significant change. The average for $1 / k_{2}$ for this set (not included in Table II) was $139 \mathrm{~s}(29 \mathrm{SD})$.

The effect of temperature on the recovery rate was examined by comparing room temperature runs and cooled runs from the midconcentration set (see Table II).

\section{Double-Exponential, Reaction-Limit Fitting Function}

Of the 172 runs, 137 were successfully fit by the double-exponential fitting function

$$
F(t)=A_{1}-A_{2} e^{-A_{3 t}}-A_{4} e^{-A s t}
$$

This function is appropriate for the case where the receptors exhibit two classes of kinetic behavior, and each process is reaction rate limited. Parameter $A_{1}$ is the final fluorescence, $A_{2}+A_{4}$ is the magnitude of the recovery, and $A_{3}$ and $A_{5}$ are the dissociation rate constants with respective percentage recoveries, $A_{2} /\left(F_{\text {pre }}-A_{1}+A_{2}\right.$ $\left.+A_{4}\right)$ and $A_{4} /\left(F_{\text {pre }}-A_{1}+A_{2}+A_{4}\right)$. All five $A_{i}$ 's are nonnegative free parameters. In a successful fit, the gradient search parameter of the fitting routine dropped by orders of magnitude, usually after only a few generations of trial functions. In a typical unsuccessful fitting attempt, the gradient search parameter remained near its starting value after many generations of trial functions.

Average values for the inverses of the rate constants and their percentage recoveries based on Eq. (4) are shown in Table II. Only 7 of the 137 fits had reduced chi-square values greater than 1.5 and are still included in the average.

Of the 137, 105 successful double exponential fits were judged to give an improved fit over the singleexponential fits. The criterion for an improved fit was that the reduced chi-square for the double-exponential fit be at least 0.05 less than for the single exponential fit. All of the 44 runs whose single-exponential fit reduced chi-squares were greater than 1.5 were successfully fit by the double exponential fitting function.

\section{Exact Fitting Function}

Of the 172 runs, 103 were successfully fit with the exact general expression of fluorescence recovery with a single dissociation rate constant:

$$
\begin{aligned}
F(t)=A_{1}-A_{2}\left\{\frac{1}{c_{1}-c_{2}}\right. & \left(c_{1} e^{c_{2}^{2} t} e r f c\left(c_{2} \sqrt{t}\right)\right. \\
& \left.\left.-c_{2} e^{c_{1}^{2} t} \operatorname{erfc}\left(c_{1} \sqrt{t}\right)\right)\right\}
\end{aligned}
$$

This function is appropriate for the case where all the receptors exhibit the same kinetic behavior but the re- 
action rates at the surface may be so fast that bulk diffusion rates may at least partially retard the recovery rate. Parameters $c_{1,2}=\sqrt{A_{4}} \pm \sqrt{A_{4}-A_{3}} . A_{1}, A_{2}$, and $A_{3}$ are the final fluorescence $F(\infty)$, the magnitude of the recovery, and the dissociation rate constant $k_{2}$ as in the single exponential fits, respectively. Parameter $A_{4}$ is the quantity $\left(k_{2} C / A\right)^{2} /(4 D)$, where $C$ and $A$ are the surfacebound and bulk concentrations, respectively, of EGF at equilibrium, and $D$ is the bulk diffusion coefficient of EGF.

Equation (5) implicitly contains two characteristic times $t_{\mathrm{bd}}$ and $t_{\text {off }}$ [4], the relative values of which determine the shape of $F(t)$ :

$$
\begin{aligned}
t_{\text {off }} & \equiv \frac{1}{k_{2}} \\
t_{\text {bd }} & \equiv \frac{l^{2}}{4 D}
\end{aligned}
$$

To see the significance of these times, it is convenient to define a parameter $l$, the depth of solution that contains the same number of solute molecules in the bulk as are adsorbed at the surface at equilibrium, per unit area. In terms of the symbols of the reaction scheme [Eqs. (1) and (2)],

$$
l \equiv C / A=[E G F \cdot R] /[E G F]
$$

Characteristic time $t_{\mathrm{bd}}$ is the average time needed (within a constant factor) for bulk diffusion through this depth $l$ toward (or away) from the surface. (Note that Thompson et al. [4] give bulk diffusion rate parameter $R_{\mathrm{BND}}=1 /$ $\left.4 t_{\mathrm{bd}}\right)$. Characteristic time $t_{\text {off }}$ is the average time that an EGF molecule remains bound to a particular receptor before dissociating.

The limit in which the $t_{\mathrm{off}}>>t_{\mathrm{bd}}$ is called the "reaction limit" by Thompson et al. [4] since the chemical kinetcs provide the rate-limiting step in the fluorescence recovery. The opposite extreme $t_{\text {off }}<<t_{\mathrm{bd}}$, in which bulk diffusion is the rate-limiting step, is called the "bulk diffusion limit." It is desirable to be in or near the reaction limit in order easily to measure the dissociation rate constant $k_{2}$ of EGF interacting with its receptor on A431 cells. Note that, in principle, the reaction limit can be approached by increasing the bulk concentration $A$ so that $l \equiv C / A \rightarrow 0$ due to saturation of receptors (since $C=$ constant at saturation).

The ratio of fitted parameters $A_{4} / A_{3}$ derived by fitting Eq. (5) to the data is equal to the ratio of characteristic times $t_{\mathrm{bd}} / t_{\mathrm{off}}$. As $A_{4} / A_{3}$ decreases, the shape of the recovery approaches the reaction limit exponential form of Eq. (3). In the other extreme, as $A_{4} / A_{3}$ increases, the shape of the recovery curve is determined more by bulk diffusion and less by dissociation kinetics. This gives $F(t)$ a very slow approach to its asymptote as $t \rightarrow$ $\infty$ (with a functional form approaching $t^{-1 / 2}$ ) and a generally longer time scale than the reaction limited form.

Average values for $t_{\mathrm{off}}=1 / k_{2}, t_{\mathrm{bd}}=l^{2} / 4 D$, and the percentage recovery are shown in Table II. Four of these 103 fits had reduced chi-square values greater than 1.5 but are included in the averages. A few of the sets of data were excluded from the average because they did not yield a reliable value for $k_{2}$. Of the 103 runs, 26 had values of $A_{4}>0.01$. This causes the ratio of $A_{4}$ to the average $A_{3}$ to exceed unity, which leads to bulk diffusion dominated recoveries. Two runs exhibited bleaching during probe illumination. This left 75 runs in the second "selected" set in Table II.

Some runs did not fit the double-exponential or the exact fitting functions. Occasionally, a run with a relatively low signal-to-noise ratio and a low reduced chisquare when fit to a single-exponential recovery curve could not be fit by the double exponential. Evidently, for these single-exponential fits, the reduced chi-square had a sharp minimum in $A_{1} A_{2} A_{3}$ space, whereas for the double-exponential fit the minimum in $A_{1} A_{2} A_{3} A_{4} A_{5}$ space was not sharply defined, and the program could not locate it in a reasonable computational time. However, when the single-exponential fitting function could not fit the data well, then the double-exponential fit could give an improved fit. This is consistent with the observation that all of the runs with poor single-exponential fits (reduced $\chi^{2}>1.5$ ) were successfully fit with double exponentials.

The exact fitting function, Eq. (5), was unable to fit as many runs as the double-exponential fitting function. Usually in an unsuccessful fit the parameters $A_{3}$ and $A_{4}$ both increased steadily with successive iterations. Presumably, no unique pair of values for these parameters could be associated with a given characteristic time in curves that were largely bulk-diffusion limited. In such a case, $A_{3}$ and $A_{4}$ both increase in a manner that maintains a constant characteristic time for bulk diffusion,

$$
t_{\mathrm{bd}}=\frac{l^{\dot{2}}}{4 D}=\frac{A_{4}}{A_{3}^{2}}
$$

Concentration effects were examined with 5 runs with a low f-EGF concentration $(\leq 5 \mathrm{n} M)$ and 21 runs with a high concentration $(\geq 10 \mathrm{nM})$ (see Table II).

\section{F-Insulin on A431 Cells}

Results from the control TIR/FRAP runs using $\mathrm{f}$ insulin in place of $f$-EGF were parameterized by curve 
fitting to the single-exponential reaction limit fitting function. The fluorescence recovery curves showed $80 \%$ reversible binding with dissociation rates greater than $0.1 \mathrm{~s}^{-1}$. This is about an order of magnitude faster than the desorption rates for specific binding of f-EGF.

\section{Free Fluorescein on A431}

When cells are exposed to free FITC (at 10 to 50 $\mathrm{n} M$ at $\mathrm{pH} 7.4$ ), visual inspection showed no green fluorescein labeling, only the usual autofluorescence.

\section{Chasing and Blocking Experiments}

Chase experiments (f-EGF followed by unlabeled EGF) were performed on 12 of the 24 f-EGF A431 samples that provided the above TIR/FRAP results. The ratio of unlabeled EGF to f-EGF was typically 50 or more. After addition of the unlabeled EGF to a sample already labeled with $\mathrm{f}$-EGF, the average fluorescence intensity dropped to about $15 \%$ of its original value within several tens of minutes. The magnitudes of fluorescence recoveries from TIR/FRAP experiments performed after the addition of the unlabeled EGF were less than 10\% of the magnitudes before addition of the unlabeled EGF. In addition, visual observation showed a clear decrease in fluorescein fluorescence after unlabeled EGF was added.

Blocking experiments (unlabeled EGF followed by f-EGF) were performed on two samples. The fluorescence intensity and magnitude of fluorescence recovery after addition of $\mathrm{f}$-EGF to the cells were similar to those in the above chasing experiments after unlabeled EGF was added. In addition, visual observation before and after addition of the f-EGF showed that there was very little fluorescein labeling of the cells.

When the chasing and blocking experiments were performed using unlabeled insulin in place of unlabeled EGF, the fluorescence from $\mathrm{f}-\mathrm{EGF}$ was unaffected by the insulin.

\section{DISCUSSION}

This paper demonstrates a new application of TIR/ FRAP to measure specific binding kinetics of a peptide hormone, EGF, to cells in culture. The technique has a number of desirable features relative to the more standard radioactive label/concentration jump methods for measuring receptor binding kinetic rates. (1) TIR/FRAP can measure faster kinetic rates, in principle, up to the bulk diffusion limit because the technique involves no solution changes; (2) it is spatially specific, because small parts of single cells can be examined rather than large groups of cells; (3) surface selectivity is based upon optical rather than chemical means; and (4) the response of the sample to a series of treatments can be followed continuously on the same culture dish.

\section{Specificity of Binding}

The term "specific" binding must be precisely defined because (1) the biochemical actions of EGF are diverse; (2) more than one class of binding constant may be present; and (3) the cell surface itself, apart from its EGF receptors, may adsorb EGF. Specific binding here is operationally defined as binding which both is saturable within the concentration range explored and is selective for the ligand at least to some extent.

The chasing and blocking experiments show that the fluorescence recovery curves from the TIR/FRAP experiments are due almost entirely to specific binding of f-EGF to the A431 cells. In chasing experiments, the addition of a large excess of unlabeled EGF to cells labeled with f-EGF and still in the presence of f-EGF caused the fluorescence of cells to drop to $15 \%$ of its prechase value. The remaining fluorescence included autofluorescence of the cell in addition to any f-EGF which remained. The chasing experiments also showed that over $90 \%$ of the TIR/FRAP fluorescence recovery magnitude is due to specifically bound f-EGF. In the blocking experiments, a concentration of f-EGF which could brightly label untreated A431 cells was not able visibly to label cells which had been previously exposed to a much higher concentration of unlabeled EGF. This observation confirms that of Haigler et al. [21] on a similar system. In the control experiment for selectivity of binding in which unlabeled insulin was used for chasing instead of the unlabeled EGF, the fluorescence was unaffected. We conclude from these chasing and blocking experiments that unlabeled EGF competes with $\mathrm{f}$-EGF for specific binding sites on our $\mathrm{A} 431$ preparation and that this binding accounts for almost all of the observed fluorescence.

Comparisons of labeled cells at low concentrations of either f-EGF or f-insulin gave further evidence of specific binding and demonstrates the high affinity of f-EGF to its receptor. Insulin and EGF are both small polypeptide hormones. However, presumably because of differences in surface charge, insulin's nonspecific binding to a biological membrane (erythrocytes) is stronger than that of EGF [11]. Therefore, the observation that nanomolar concentrations of f-EGF brightly label A431 cells whereas similar concentrations of f-insulin gave no visible labeling (in experiments not previously discussed), combined with the fact that A431 cells are known 
to overexpress the EGF receptor, further suggests that almost all of the f-EGF binding is to its receptor.

Fluorescence of labeled cells at various concentrations of f-EGF confirms an affinity consistent with published dissociation equilibrium constants in the nanomolar range $[14,16,27]$.

\section{Kinetic Rates}

The single-exponential fitting function rate of 0.007 $\mathrm{s}^{-1}\left(1 / k_{2}=140 \mathrm{~s}\right)$ and the exact fitting function rate of $0.012 \mathrm{~s}^{-1}\left(1 / k_{2}=85 \mathrm{~s}\right)$ are within the range of published values (see Table I) for the dissociation rate constant of ${ }^{125} \mathrm{I}$-EGF interacting with EGF receptors.

The following sections address whether any of the fitting functions are appropriate for determining the dissociation rate constant of the hormone-receptor interaction. The first consideration is (a) whether the experiments were in the reaction limit, in which case bulk diffusion can be ignored. Then two other processes which could influence the fluorescence recovery rate are considered. These are (b) hindered access of cell surface to $\mathrm{f}-\mathrm{EGF}$ and (c) lateral diffusion of hormone-receptor complexes in the cell membrane.

\section{Bulk Diffusion}

Here we discuss whether the TIR/FRAP experiments were indeed in or near the reaction limit, so that $F(t)$ is significantly influenced by the value of $k_{2}$. The condition for the fluorescence recovery to be in the reaction limit is $t_{\text {off }}>>t_{\mathrm{bd}}$ as discussed under Results. However, the definition given for $t_{\mathrm{bd}}\left(=l^{2} / 4 D\right)$ is appropriate only if the lateral $(x-y)$ dimension of the illuminated region is very large compared to $l=C / A$ so that only bulk diffusion in the z-direction is important. In an actual experiment the region of illumination is finite, which may give rise to a smaller characteristic distance and thereby a shorter $t_{\mathrm{bd}}$. This occurs if the dimension of the illuminated region is smaller than $l$. In that case, a new characteristic distance, $l^{\prime}$, can be estimated semiqualitatively by following the same principle as for estimating $l$ in the one-dimensional case, i.e., determining the volume of bulk $V$ that contains the same number of molecules as does the surface in the illuminated area $s$. If that volume has characteristic dimension $l^{\prime}$ such that $l^{\prime 3}=V$, then equating the numbers of molecules gives

$$
A l^{\prime 3}=C s
$$

Assuming that $\mathrm{f}-\mathrm{EGF}$ has unhindered access to the cell surface adjacent to the substrate, then the time for bulk diffusion over distance $l^{\prime}$ is

$$
\imath_{\mathrm{bd}}=\frac{l^{\prime 2}}{4 D}=\frac{(C s / A)^{2 / 3}}{4 D}
$$

where $D$ is the bulk diffusion coefficient, $5 \times 10^{-7} \mathrm{~cm}^{2} /$ s.

From Eq. (11), we can estimate $t_{\mathrm{bd}}$ and compare it to the actual characteristic time $\tau$ for fluorescence recovery in order to determine if the experiments were in the reaction limit. A recovery cannot occur in a time shorter than the bulk diffusion-limited time; therefore, if $\tau \approx t_{\mathrm{bd}}$, the kinetic process is in the bulk diffusion limit. If $\tau>>t_{\mathrm{bd}}$, the process is in the reaction limit. Under our experimental conditions, the bulk concentration $A$ was in the range of $1-50 \mathrm{nM}$ and $s$ was about 100 $\mu \mathrm{m}^{2}$. Assuming the worst case where all receptors are occupied, $C=3 \times 10^{11} \mathrm{~cm}^{-2}$. For $A=10 \mathrm{nM}$, this gives $l^{\prime}=0.0035 \mathrm{~cm}$ and a time for bulk diffusion $t_{\mathrm{bd}}$ $=6 \mathrm{sec}$. The time $\tau$ for a typical fluorescence recovery, as fit by the single-exponential function, was about 140 $\mathrm{s}\left(\right.$ rate, $0.007 \mathrm{~s}^{-1}$ ) in our experiments. This gives a ratio of $t_{\mathrm{bd}}$ to the observed recovery time of approximately 0.04. Single-exponential fits of simulated curves [generated by the exact function form of Eq. (5) with both $1 / k_{2}$ and $t_{\mathrm{bd}}$ as known input variables] showed that for ratios of 0.01 and 0.10 , the single-exponential rate would underestimate $k_{2}$ by 10 and $22 \%$, respectively. This indicates that our experiments were near, but not completely in, the reaction limit and therefore the exact fitting function results should be used. A fit of our typical data to the exact function gives a rate of $k_{2}=0.012 \mathrm{~s}^{-1}$. The ratio of $t_{\mathrm{bd}}$ to the observed $1 / k_{2}$ then gives the ratio of 0.07 , suggesting that the single-exponential rate underestimates $k_{2}$ by about 20\%. This is consistent (considering the estimates involved in determining the ratio) with the single-exponential result of $0.007 \mathrm{~s}^{-1}$ being about $40 \%$ below $0.012 \mathrm{~s}^{-1}$.

The exact fitting procedure yields a value of $t_{\mathrm{bc}}$ of $\sim 19 \mathrm{~s}$ (from the second "selected" group in Table II), which is reasonably close to the estimated $t_{\mathrm{bd}}$ of $6 \mathrm{~s}$, considering the crude approach in the estimate.

Single-exponential fits as a function of concentration showed that the recovery rate increased with concentration, reaching a maximum of about $0.0075 \mathrm{~s}^{-1}$. The increasing rate would be expected for experiments not completely in the reaction limit; as the bulk concentration increases, the limit imposed by bulk diffusion should become less and less important and the recovery rate should approach the actual chemical kinetics rate $k_{2}$. However, the maximum rate observed in the high-con- 
centration cases should agree with the $k_{2}$ rate derived at lower concentrations from the exact fitting function, i.e., $0.012 \mathrm{~s}^{-1}$. In addition, the exact fitting function rate for $k_{2}$ should be independent of concentration. But the results showed that this rate increased with concentration. These two problems could be due to biological variability insufficiently averaged out by the number of runs performed or a multiplicity of binding classes with different binding rates and degrees of saturation as the concentration is varied. Note, however, in Table II that $t_{\mathrm{bd}}$ as derived from the exact fitting function did decrease with increasing concentration $A$.

The double-exponential reaction limit fitting function generally did not give much better fits than the exact fitting function. Therefore since the experiments were not completely in the reaction limit and because the exact fitting function has fewer free parameters, it is not advantageous to use a two-affinity state model of binding to explain the results.

\section{Accessibility}

The evanescent field of TIR extends about $100 \mathrm{~nm}$ into the aqueous buffer, so that only fluorescence on the cell surfaces in close proximity to the glass can be excited. Access by the bulk hormone to binding sites in this surface-proximal region may be restricted. This possibility was examined in control experiments using f-insulin with A431 cells. The fluorescence recovery in these experiments is due to unbleached f-insulin in solution diffusing underneath the cell and replacing bleached f-insulin adsorbed to the cell and bare glass and in the layer of solution between the cell and the glass. Singleexponential fits of these fluorescence recovery curves gave fluorescence recovery rate constants greater than $0.1 \mathrm{~s}^{-1}$. Thus it takes $10 \mathrm{~s}$ or less for $\mathrm{f}$-insulin to diffuse underneath the A431 cell.

This result agrees with the following visual observation. If the laser intensity was increased high enough during visual observation of f-insulin with A431 cells, the central portion of an A431 cell could be made to turn dark. If the laser illumination was then stopped for about $10 \mathrm{~s}$, upon resumed illumination the cells would appear bright again. The darkening occurred because the rate of photobleaching increased beyond the rate at which diffusion replaced bleached fluorophore with unbleached ones. Thus it takes f-insulin about $10 \mathrm{~s}$ to diffuse in between the cell and the glass substrate. Since insulin and EGF are small polypeptide hormones of similar molecular weight, it is reasonable to assume that they would have similar access to the cell surface adjacent to the substrate.
For unrestricted access, the time to diffuse over an average distance of $5 \mu \mathrm{m}$ with a free bulk diffusion coefficient of $D=5 \times 10^{-7} \mathrm{~cm}^{2} / \mathrm{s}$ into the space between a A431 cell and the coverslip should be about 0.5 $\mathrm{s}$. This is shorter than the 10 -s recovery time actually measured for f-insulin, suggesting that access may be slightly restricted. Alternatively, the $10-\mathrm{s}$ recovery time could reflect the nonspecific.adsorption time of $\mathrm{f}$-insulin to glass and cell surface. Fulbright [11] found that the typical time that $f$-insulin stays nonspecifically bound to red blood-cell membrane is of the order of seconds.

Although it appears that access to the cell surface adjacent to the substrate may be hindered, the recovery rate of $\mathrm{f}$-insulin interacting with A431 cells is faster than the rate for $\mathrm{f}-\mathrm{EGF}$. Therefore, the fluorescence recovery of f-EGF binding to A431 cells does not seem to be rate limited by such hindered access.

\section{Lateral Diffusion}

Experimental measurements and theoretical estimates were performed to check the possibility that the fluorescence recovery rate might be influenced by lateral diffusion of unbleached EGF-receptor complexes from parts of the cell beyond the evanescent wave, as shown schematically in Fig. 4.

In control experiments, cells were fixed in $4 \%$ formaldehyde for $30 \mathrm{~min}$ in order to inhibit lateral diffusion of receptors. Single-exponential recovery rates from TIR/ FRAP experiments performed on these samples $\left(1 / k_{2}=\right.$ $135 \mathrm{~s} ; 35 \mathrm{~s} \mathrm{SD})$ showed no significant difference from the results in Table II.

In theoretical estimates, a simulated recovery curve which includes the effect of lateral diffusion (see Appendix 3) was curve fit using the same fitting functions that were used on the data. The desorption rate calcu-

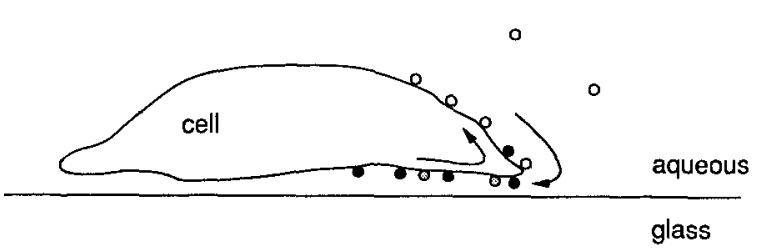

Fig. 4. Diagram showing how lateral diffusion of hormone-receptor complexes at the cell membrane could replace bleached fluorophore with unbleached fluorophore, thus contributing to the fluorescence recovery in a TIR/FRAP experiment. The evanescent field of TIR excitation can excite and photobleach only fluorophores on the cell surface adjacent to the glass substrate. The circles are fluorophores as follows: open circles are unbleached and unexcited, shaded circles are unbleached and excited, and filled circles are bleached. 
lated by the fitting functions was then compared to the input desorption rate of the simulated recovery curve. This gives an estimate as to how much the fitting function desorption rates could differ from the true desorption rate in the presence of lateral diffusion. As discussed in Appendix 3, for reasonable values of receptor diffusion coefficients and assumptions about the distance of diffusion, the theoretical estimates show that lateral diffusion is likely to have little or no effect on the fluorescence recovery rates.

\section{Significance of Dissociation Rate Constant}

Our TIR/FRAP results for the $1 / k_{2}$ mean dissociation time (Table II) are at the low end of the range of reported results from ${ }^{125}$ I-EGF binding assays (see Table I).

The relevance of these results to the native EGF interaction depends on the effect of the fluorescent label and on the effect of fixing the cells. Concerning the fluorescent label, these results and others [21,27] have shown that f-EGF competes with native EGF for specific binding sites. Haigler et al. [21] also showed that f-EGF stimulates DNA replication in human fibroblasts. Concerning the fixing, the TIR/FRAP results showed no difference between cells fixed at 0.2 or $4 \%$ formaldehyde and unfixed cells. This observation agrees with the previous result that fixing in $0.2 \%$ formaldehyde did not alter affinity [14] and that cells fixed in 3\% formaldehyde appeared the same as unfixed cells when stained with f-EGF [21].

The large standard deviation in our results has a number of potential sources. As explained earlier, each TIR/FRAP run monitored a portion of a single cell, whereas ${ }^{125}$ I-EGF dissociation assays average over hundreds of thousands of cells. Therefore, the TIR/FRAP results may demonstrate cell-to-cell variability. In addition, using A431 cells at different stages of growth may have caused variability. Some samples had patches of fewer than 10 cells, whereas other samples were close to confluent. Kanuer et al. [16] found in ${ }^{125}$ I-EGF binding assays that variations in measured rate constants could be kept to less than $10 \%$ by following protocols for cell culture and sample preparation that are stricter than those used in these TIR/FRAP experiments. If these two effects were responsible for the variability, then TIR/FRAP runs performed at the same place should show less variability than runs performed at different places and on different samples. However, TIR/FRAP runs at the same place showed variability only slightly less than all runs combined. Therefore it seems likely that some samples may have changed with time, possibly due to changes in cell morphology during the fluorescence recovery. If the cell membrane moved with respect to the substrate, this would change the fluorescence signal due to the distance dependence of TIR excitation.

These results are consistent with all models of hormone/receptor interaction mentioned under EGF/Receptor Binding Kinetics (Introduction). In part, this is because the simple model we use is a part of the single-affinity state models and also can apply to $90-95 \%$ of the receptors in the two-affinity state model. In addition, the large variation in our results makes distinction between the models difficult.

Our results allow a number of semiqualitative conclusions to be drawn regarding the behavior of a typical hormone at the cell surface. An f-EGF molecule typically stays bound to an EGF-receptor for $t_{\text {off }} \approx 100 \mathrm{~s}$. No shorter time components appear to be present, although TIR/FRAP could have detected them if they existed. The fact that $t_{\text {off }}$ is generally two to five times longer than the $t_{\mathrm{bd}}$ for our particular ratio C/A of surfaceto-bulk f-EGF concentrations suggests that when a typical hormone dissociates from a receptor, it ventures far enough into the bulk so that it is unlikely to rebind to the same receptor. (Note, however, that the C/A ratio and the corresponding $t_{\text {bd }}$ may be different for other cell types and bulk EGF concentrations.) Since the 100 -s $t_{\text {off }}$ time is shorter than the 500-s time reported for endocytosis $[16,19]$, a typical bound f-EGF is likely to dissociate from the first receptor it finds before the complex has a chance to become internalized.

Since the association rate $k_{1}$ equals $k_{2} / K_{\mathrm{D}}$ where $\mathrm{K}_{\mathrm{D}}$ is the previously measured equilibrium dissociation constant, one can infer a value of $k_{1}$ from a measured value of $k_{2}$. Using $K_{\mathrm{D}}=5 \mathrm{n} M$, which is in the range of published values $[1,12,16,18,20]$, and a value of $k_{2}$ $=0.01 \mathrm{~s}^{-1}$ as measured here, we derive that $k_{1}=2 \times$ $10^{6} \mathrm{~s}^{-1} \mathrm{M}^{-1}$. The maximum possible theoretical $k_{1}$, for which every diffusive encounter between a three-dimensional dissolved ligand and a two-dimensional immobilized receptor leads to a successful binding (assuming a target radius of $5 \AA$ and a bulk diffusion coefficient $D$ of $5 \times 10^{-7} \mathrm{~cm}^{2} / \mathrm{s}$ ), is $k_{1} \cong 1 \times 10^{8} \mathrm{~s}^{-1} M^{-1}$ (derived from the exact solution for "Weber's disc" [30]). Therefore, we can conclude that no more than $2 \%$ of the EGF/ receptor encounters in our system are successful.

If the receptor-containing target was really a flat, infinite plane, and the bulk solution was really infinite in depth as assumed in our model, then we could also conclude from the nearly (but not completely) reaction rate-limited state of the process that the first dissociation would usually not be followed by rebinding to any other receptor. However, in an actual biological organism, the 
receptor-containing cell surfaces partially surround free bulk volumes which are far from infinite in extent. Therefore, assuming that the in vivo concentration ratio $\mathrm{C} / \mathrm{A}$ is not too different from that in our experiments, several association/dissociation events at different receptors are likely, followed ultimately by internalization.

In summary, we have shown that TIR/FRAP can successfully measure dissociation rates of ligands from cell surface targets on intact cells in culture.

\section{APPENDIX 1: PRISMLESS TIR FLUORESCENCE MICROSCOPY}

A description and demonstration of epiillumination (i.e., through the objective) or "prismless" TIR are given by Stout and Axelrod [23]; a new variant is used here. The technique is based on the correspondence of radial position of a beam at the objective's back focal plane with its angle of emergence toward the sample plane (see Fig. 1). All light rays converging upon the same point in the back focal plane propagate parallel to one another on the object side of the lens. The maximum possible value of the angle of propagation with respect to the optical axis is given by the numerical aperture, $N A=$ $n_{\mathrm{g}} \sin \theta_{\max }$, where $n_{\mathrm{g}}$ is the refractive index of the glass substrate at the sample plane. For the glass/water interface, incidence angles greater than the critical angle for total internal reflection are possible if $N A>n_{\mathrm{w}}=1.33$. Therefore an objective with $N A=1.4$ was used.

Ensuring that the laser beam is focused at a particular off-axis radial position at the objective's back focal plane can be accomplished by locating an "equivalent" back focal plane upbeam from the microscope. ("Equivalent" back focal plane means that light focused to a point in this plane is also focused to a point in the objective's back focal plane.) For our Leitz Diavert epifluorescence microscope, an equivalent back focal plane exists at about $10 \mathrm{~cm}$ upbeam from the field diaphragm (see Fig. 1). Mirrors and lenses direct the laser beam to focus at an off-axis point at the equivalent back focal plane and then pass through the center of the field diaphragm. In order to keep the beam diameter reasonably small at the field diaphragm (thereby ensuring a small region of illumination on the sample), the focal length of this lens should be $>100 \mathrm{~mm}$.

TIR is achieved by increasing the radial position of the point in the equivalent back focal plane while viewing a sample containing a surface-bound fluorophore of one emission color and a solute fluorophore of another emission color. For small radial positions in the equivalent back focal plane, transmitted light excites fluores- cence in the bulk solution which is easily distinguished by its color. As the radial position is increased, the angle of incidence at the glass/water interface becomes large enough for TIR, resulting in excitation of only the distinct color of surface fluorescence.

\section{APPENDIX 2: PHOTOBLEACHING DURING ILLUMINATION INCREASES THE RECOVERY RATE}

This appendix shows the effect of photobleaching during probe illumination on the measured fluorescence recovery rate in a TIR/FRAP experiment performed on a system with reversible chemical kinetics at a surface. We assume that the chemical process $(A+B \rightleftharpoons C$ in the same notation as elsewhere in this paper) is in the "reaction limit."

The differential equation describing the surface concentration of fluorescent ligand-receptor complexes in the reaction limit is

$$
\frac{\partial C_{\mathrm{fl}}}{\partial t}=A_{\mathrm{fl}} B k_{1}-\gamma C_{\mathrm{fl}}-k_{2} C_{\mathrm{fl}}
$$

where $\gamma$ is the rate of conversion of fluorescent ligand to bleached ligand at the surface due to photobleaching with the laser at probe illumination level. In the reaction limit $A_{\mathrm{bl}}(t)=0$. Therefore, $A_{\mathrm{fl}}=A$, and the steadystate surface concentration is

$$
C_{\mathrm{fl}}(\infty)=\frac{A B k_{1}}{\left(\gamma+k_{2}\right)}
$$

Therefore after a photobleaching pulse, the concentration (and hence fluorescence) is given by

$$
C_{\mathrm{fl}}(t)=\frac{A B k_{1}}{\left(\gamma+k_{2}\right)}-\alpha e^{-\left(\gamma+k_{2}\right) t}
$$

Thus the fluorescence recovery rate is greater than the off rate $k_{2}$.

\section{APPENDIX 3: EFFECT OF LATERAL DIFFUSION OF SPECIFIC BINDING SITES}

Consider a cell adhered to a coverslip on which hormone/receptor complexes in membrane adjacent to the coverslip are photobleached in a TIR/FRAP experiment. Fluorescence recovery here is assumed to occur by two mechanisms: (i) chemical reaction kinetics of exchange between a bulk-dissolved and surface-bound hormone, as usual; and (ii) lateral diffusion of com- 
plexes between the photobleached region of the membrane and the unphotobleached regions beyond the evanescent field. For simplicity, this situation is modeled by one-dimensional diffusion in which $C_{\mathrm{bl}}(x>0$, $t=0)=1$ and $C_{\mathrm{bl}}(x<0, t=0)=0$, where $C_{\mathrm{bl}}(x, t)$ is the concentration of bleached complexes and the photobleaching pulse occurred at $t=0$. The equation describing the time evolution of the concentration of bleached complexes is

$$
\frac{\partial C_{\mathrm{bl}}(x, t)}{\partial t}=D_{\mathrm{C}} \frac{\partial^{2} C_{\mathrm{bl}}(x, t)}{\partial x^{2}}-k_{2} C_{\mathrm{bl}}(x, t)
$$

where $D_{\mathrm{C}}$ is the lateral diffusion coefficient of the hormone-receptor complex. For the initial conditions $C_{\mathrm{bl}}$ $(x>0, t=0)=1$ and $C_{\mathrm{bl}}(x<0, t=0)=0$, the solution to Eq. (A4) is

$$
C_{\mathrm{bl}}(x, t)=\frac{1}{2} e^{-k 2 t} e r f c\left(\frac{-x}{\sqrt{4 D_{\mathrm{C}}}}\right)
$$

Simulated fluorescence recoveries were calculated on computer on the basis of Eq. (A5) by integrating over a window of $x$ values. This region extended from $x=$ 0 (the boundary between bleached and unbleached membrane) to $x=5 \mu \mathrm{m}$ (within the bleached region). This fluorescence-collection window simulates the portion of a cell next to bare glass as described under Materials and Methods. The input lateral diffusion coefficient $D_{\mathrm{C}}$ was $4 \times 10^{-10} \mathrm{~cm}^{2} / \mathrm{s}[28,29]$. The overestimate that $100 \%$ of the receptors were mobile was made in order to estimate the largest possible effect that lateral diffusion could have on the fluorescence recovery curve.

Now we examine how the kinetic parameters as determined by our fitting procedures might be affected by the presence of lateral diffusion in the simulated curves, for the range of single kinetic parameters we have derived from our data. For a simulated recovery curve with an input dissociation rate of $0.0070 \mathrm{~s}^{-1}$, the single-exponential fit gave $k_{2}=0.0086 \mathrm{~s}^{-1}$, and the exact fit gave $0.0097 \mathrm{~s}^{-1}$ with a $t_{\mathrm{bd}} / t_{\text {off }}=0.01$. For a simulated recovery curve with an input dissociation rate of 0.010 $\mathrm{s}^{-1}$, the single-exponential fit gave $k_{2}=0.0118 \mathrm{~s}^{-1}$, and the exact fit gave $k_{2}=0.0128 \mathrm{~s}^{-1}$ with a $t_{\mathrm{bd}} / t_{\text {off }}=$ 0.003 . Thus, at worst, the $k_{2}$ value from curve fitting is $39 \%$ too high because of the influence of receptor lateral diffusion.

It should be emphasized that this is an upper limit on the error. The value chosen for $D_{\mathrm{C}}$ is probably high because it is for spot photobleaching results which measure diffusion over shorter distances $(\approx 1 \mu \mathrm{m})$, which is generally less restricted than the much longer-range diffusion required to affect the fluorescence recovery in these TIR/FRAP experiments [25]. Also, the estimate assumes that $100 \%$ of the receptors are mobile. Reported results are that only $60 \%$ are mobile $[28,29]$. This would diminish the effect of lateral diffusion, since then $40 \%$ of the receptors' fluorescence could recover solely via kinetics. In addition, many of the TIR/FRAP experiments were done on cells fixed in $0.2 \%$ formaldehyde. This would further inhibit lateral diffusion.

Using the more reasonable estimates of $D_{\mathrm{C}}=1 \times$ $10^{-10} \mathrm{~cm}^{2} / \mathrm{s}$ [25] and $60 \%$ of the receptors mobile, another simulated recovery curve was produced and curve fit. For a simulated recovery curve with an input dissociation rate of $0.0070 \mathrm{~s}^{-1}$, the single exponential fit gave $k_{2}=0.0075 \mathrm{~s}^{-1}$, and the exact fit gave $k_{2}=$ $0.0077 \mathrm{~s}^{-1}$ with a $t_{\mathrm{bd}} / t_{\text {off }}=0.0006$. In this case, the fitting functions overestimate the dissociation rate by no more than $10 \%$.

This model assumes an infinite diameter for the cell. The validity of this assumption was checked by calculating the concentration in Eq. (33) at a distance of $5 \mu \mathrm{m}$ from the border between bleached and unbleached regions, using $k_{2}=0.0070 \mathrm{~s}^{-1}$ and $D_{\mathrm{C}}=4 \times 10^{-10}$ $\mathrm{cm}^{2} / \mathrm{s}$, and comparing it to the concentration with no lateral diffusion. The result was that the difference between the two recovery curves as a function of time, normalized by the initial concentration, was always less than $3 \%$. The difference between the two recovery curves is so small because the characteristic time for lateral diffusion over a distance of $5 \mu \mathrm{m}$ is longer than the 1/e kinetic recovery time. Therefore, the infinite diameter assumption is valid since in the region of observation used in the experiments, diffusion from only the nearest border would affect the fluorescence recovery curve.

\section{ACKNOWLEDGMENTS}

We thank Robert Fulbright for help with the computer software, Keith Shaw for help in maintaining the equipment, Sharada Kumar for technical and clerical support, Dr. David Johnson for sharing his EGF labeling protocols with us, Dr. George Barisas for suggesting the butanol extraction method, and Drs. Joseph Schlessinger and Nancy Thompson for their useful comments on surface chemical kinetics. This project was supported by Grants NIH 14565 and NSF DMB-8805296, a University of Michigan Rackham Faculty Grant to D.A., and a University of Michigan Rackham Graduate Fellowship to E.H. 


\section{REFERENCES}

1. G. Carpenter and S. Cohen (1979) Annu. Rev. Biochem. 48, 193216.

2. F. Bellot, W. Moolenaar, R. Kris, B. Mirakhur, I. Verlaan, A. Ullrich, J. Schlessinger, and S. Felder (1990) J. Cell Biol. 110, 491-502.

3. M. Benveniste, E. Livneh, J. Schlessinger, and Z. Kam (1988) J. Cell Biol. 106, 1903-1909.

4. N. L. Thompson, T. P. Burghardt, and D. Axelrod (1981) Biophys. J. 33, 435-454.

5. D. Axelrod, E. H. Hellen, and R. M. Fulbright (1991) in J. Lakowicz (Ed.), Fluorescence Spectroscopy, Vol. 3. Biochemical Applications, Plenum Press, New York (in press).

6. T. P. Burghardt and D. Axelrod (1981) Biophys. J. 33, 455-468.

7. R. D. Tilton, C. R. Robertson, and A. P. Gast (1990) J. Colloid Inter. Sci. 137, 192-203.

8. C. L. Poglitsch and N. L. Thompson (1990) Biochemistry 29, 248-254.

9. E. Kalb, J. Engel, and L. K. Tamm (1990) Biochemistry 29, $1607-1613$.

10. R. M. Zimmermann, C. F. Schmidt, and H. E. Gaub (1990) J. Colloid Inter. Sci. 139, 268-280.

11. R. M. Fulbright (1991) Ph.D. thesis, University of Michigan, Ann Arbor.

12. J. Schlessinger (1986) J. Cell Biol. 103, 2067-2072.

13. J. Schlessinger (1988) Biochemistry 27, 3119-3123.

14. T. Kawamoto, J. D. Sato, A. Le, J. Polikoff, G. H. Sato, and J. Mendelsohn (1983) Proc. Natl. Acad. Sci. USA 80, 1337-1341.

15. A. C. King and P. Cuatrecasas (1982) J. Biol. Chem. 257, 30533060 .
16. D. J. Knauer, H. S. Wiley, and D. D. Cunningham (1984) $J$. Biol. Chem. 259, 5623-5631.

17. H. S. Wiley (1988) J. Cell Biol. 107, 801-810.

18. K. H. Mayo, M. Nunez, C. Burke, C. Starbuck, D. Lauffenburger, and C. R. Savage, Jr. (1989) J. Biol. Chem. 264, 1783817844.

19. C. M. Waters, K. C. Oberg, G. Carpenter, and K. A. Overholser (1990) Biochemistry 29, 3563-3569.

20. A. C. Myers, J. S. Kovach, and S. Vuk-Pavlovic (1987) J. Biol. Chem. 262, 6494-6499.

21. H. Haigler, J. F. Ash, S. J. Singer, and S. Cohen (1978) Proc. Natl. Acad. Sci. USA 75, 3317-3321.

22. J. Schlessinger, A. B. Schreiber, A. Levi, I. Lax, T. A. Libermann, and Y. Yarden (1983) CRC Crit. Rev. Biochem. 14, 93111.

23. A. L. Stout and D. Axelrod (1989) Appl. Opt. 28, 5237-5242.

24. E. H. Hellen and D. Axelrod (1990) Rev. Sci. Instrum. 61, 37223725 .

25. T. D. Giugni, D. L. Braslau, and H. T. Haigler (1987) J. Cell Biol. 104, 1291-1297.

26. P. R. Bevington (1969) Data Reduction and Error Analysis for the Physical Sciences, McGraw-Hill, New York, Chap. 11.

27. R. C. Chatelier, R. G. Ashcroft, C. J. Lloyd, E. C. Nice, R. H. Whitehead, W. H. Sawyer, and A. W. Burgess (1986) EMBO J. 5, 1181-1186.

28. G. M. Hillman and J. Schlessinger (1982) Biochemistry 21, 16671672.

29. E. Livneh, M. Benveniste, R. Prywes, S. Felder, Z. Kam, and J. Schlessinger (1986) J. Cell Biol. 103, 327-331.

30. J. Crank (1975) The Mathematics of Diffusion, 2nd ed., Oxford, New York, pp. 42-43. 